
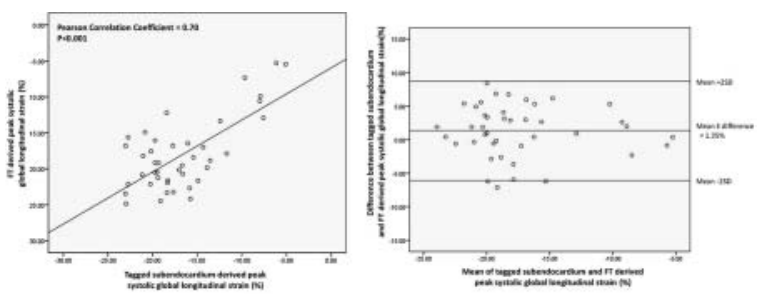

Figure 1

tagging analysis for any strain parameter other than mid-left ventricular whole slice circumferential strain. In an adult study of patients with dilated cardiomyopathy (DCM) and healthy controls, we sought to validate the FT method (TomTec Imaging systems, Munich) against spatial modulation of magnetisation (SPAMM) tissue tagging analysis (Cardiac Image Modelling Package (CIMTag2D), University of Auckland) for the computation of long axis function.

Methods We compared measures of global longitudinal strain from the horizontal long axis view using the two techniques in 45 patients (mean age $44 \pm 14$ years, male 63\%). Normal healthy adults $(n=35)$ were identified from an ongoing prospective, observational research study examining the effects of living kidney donation on cardiovascular structure and function (NCT01028703). Consecutive DCM patients $(n=10)$ were identified after undergoing myocardial tagging for clinically indicated scans. Retrospective off-line analysis was performed on matched tagged and SSFP slices by two independent blinded observers (WEM and RJT). After manually drawing endocardial borders in the end-diastolic frame for each image, the FT software automatically propagated the contour and followed its features (brightness gradient at the tissuecavity interface, dishomogeneties of the tissue, spatial coherence) throughout the remainder of the cardiac cycle to compute longitudinal strain parameters.

Results Longitudinal strain (Ell). Peak systolic FT-Ell (-18.1 $\pm 5.0 \%)$ correlated most strongly with CIMTag-Ell values derived from the subendocardium $(-16.7 \pm 4.8 \%)$ with a Pearson's correlation coefficient of $0.70(p<0.001$; figure $1 \mathrm{~A})$. A Bland Altman plot (figure 1B) showed good agreement with only a small systematic overestimation from FT (mean difference $1.3 \pm 3.8 \%, p=0.03$ ). Whilst in DCM patients peak systolic global Ell values were not significantly different between the two techniques $(-9.7 \pm 4.5 \%$ vs $-8.8 \pm 3.9 \%$, $\mathrm{p}=0.44$ ), among healthy controls there was a small but significant difference in Ell values between FT and tagging image analysis $(-19.5 \pm 3.5 \%$ vs $-18.0 \pm 3.5 \%, p=0.04$; figure 2$)$.

\title{
VALIDATION OF MAGNETIC RESONANCE FEATURE TRACKING FOR LONGITUDINAL SYSTOLIC AND DIASTOLIC STRAIN CALCULATION WITH SPATIAL MODULATION OF MAGNETISATION IMAGING ANALYSIS
}

W E Moody, ${ }_{1}^{1}$ R J Taylor, ${ }^{2}$ N C Edwards, ${ }^{1}$ C D Chue, ${ }^{3}$ F Umar, ${ }^{2}$ C J Ferro, ${ }^{1}$ J N Townend, ${ }^{1}$ F Leyva, ${ }^{2}$ R P Steeds ${ }^{1}{ }^{1}$ University Hospital Birmingham NHS Foundation Trust and Birmingham Cardio-Renal Group, University of Birmingham; ${ }^{2}$ University Hospital Birmingham NHS Foundation Trust and University of Birmingham; ${ }^{3}$ City and Sandwell NHS Foundation Trust and Cardio-Renal Group, University of Birmingham

doi:10.1136/heartjnl-2013-304019.101

Introduction Feature-tracking (FT) analysis offers a novel, fast and practicable method to calculate strain from routinely acquired steady state free precession (SSFP) images without the need to perform additional tagged sequences. There is no validation of this technique, however, against a reference standard myocardial

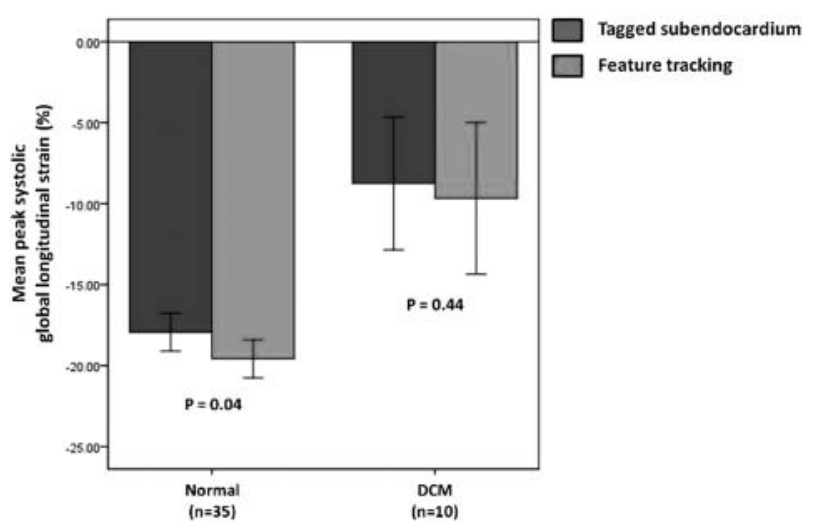

Figure 2 
Longitudinal strain rate (SR) There was good agreement between the two techniques for peak systolic global longitudinal SR values but with a small tendency towards higher FT values as compared with tagging (mean difference $0.09 \pm 0.261 / \mathrm{s}, \mathrm{p}=0.04 ; \mathrm{r}=0.64, \mathrm{p}<0.001$ ). The weakest correlation between the two techniques was for early diastolic global longitudinal SR but even this relationship was still highly significant (mean difference $0.09 \pm 0.261 / \mathrm{s} ; \mathrm{r}=0.42, \mathrm{p}=0.007$ ).

Reproducibility testing Intraobserver and interobserver variability for FT-Ell analysis was small $(-0.49 \pm 1.83 \%$ and $0.22 \pm 1.13 \%$, respectively).

Timed analysis The average time taken for post-processing strain analysis using FT software was significantly less than that required for CIMTag (5.9 $\pm 0.8 \mathrm{~min}$ vs $23.2 \pm 3.5 \mathrm{~min}, \mathrm{p}<0.0001)$.

Conclusions FT based assessment of longitudinal strain correlated highly with values derived from tagged images in a population with a wide range of left ventricular function. Furthermore, FT can be performed without the need for additional imaging and lengthy postprocessing times. 